\title{
Search for lepton flavour violating decays of the Higgs boson with CMS
}

\author{
Prasanna Kumar Siddireddy* \\ University of Notre Dame \\ E-mail: psiddire@nd.edu
}

Recent results from direct searches for lepton flavour violating decays of the Higgs boson are presented. The searches presented include lepton flavour violating (LFV) decays of Higgs(125) boson along with neutral heavy Higgs LFV decays. These searches are done with $35.9 \mathrm{fb}^{-1}$ of data collected by the CMS detector at the LHC in 2016 at a center-of-mass energy of $13 \mathrm{TeV}$.

European Physical Society Conference on High Energy Physics - EPS-HEP2019 -

10-17 July, 2019

Ghent, Belgium

* Speaker.

${ }^{\dagger}$ A footnote may follow. 


\section{Introduction}

In the standard model (SM), the Higgs mechanism explains electroweak symmetry breaking and predicts the existence of a new massive particle, the Higgs boson. During Run I at the LHC the discovery of the Higgs boson was announced by the ATLAS and CMS collaborations $[1,2]$ with the most significant excesses seen in ZZ, $\gamma \gamma$, and WW decay channels. Analysis of Higgs couplings by CMS and ATLAS have set an upper limit at 95\% confidence level (CL) on beyond the standard model (BSM) Higgs decays of $34 \%$ [3], providing us with ample room to detect exotic decays. The exotic decays could provide a portal for us to study the BSM Higgs sector. In Run II, there are many analysis focusing on the exotic decay of Higgs boson and in this document we briefly describe the search for LFV decays of the Higgs boson and neutral heavy Higgs bosons. The CMS detector is described in detail in [4].

\section{Lepton flavour violating decays of Higgs(125)}

In SM, LFV decays of the Higgs boson are forbidden. However, several BSM models such as Two Higgs Doublet, Randall-Sundrum, Composite Higgs models allow LFV decays of Higgs boson. A search for $H \rightarrow \mu \tau$ and $H \rightarrow e \tau$ with $35.9 \mathrm{fb}^{-1}$ data collected by CMS at $13 \mathrm{TeV}$ centerof-mass energy has been performed [5]. The $\tau$ lepton in the final state can decay both hadronically as well as leptonically. Final states with two same-flavor leptons are not studied due to large Drell-Yan (DY) background. So, the channels that are probed are $H \rightarrow \mu \tau_{h}, H \rightarrow \mu \tau_{e}, H \rightarrow e \tau_{h}$, $H \rightarrow e \tau_{\mu}$.

Each of the channels is divided into four categories to enhance different Higgs production modes. The channels are divided based on number of jets in the event with $p_{T}>30 \mathrm{GeV}$ and $|\eta|<4$.7. The 0 -jet and 1 -jet categories target Gluon Gluon Fusion production mode with no jets and an Initial State Radiation respectively. Based on the invariant mass of the two jets $\left(M_{j j}\right)$, the 2-jet category is further divided into two categories. Gluon Gluon Fusion production mode is dominant in events with low $M_{j j}$, while Vector Boson Fusion is the dominant production mode in events with high $M_{j j}$.

DY, $t \bar{t}$, and misidentified lepton backgrounds are the dominant backgrounds in all the channels. DY background is dominant in 0-jet category and $t \bar{t}$ background is dominant in 2-jet category. For 1-jet category, DY background is dominant in hadronic channels while for leptonic channels $t \bar{t}$ background is dominant. Monte Carlo (MC) simulations are used for estimating both these backgrounds. The misidentified background, where a jet is misidentified as a lepton comes predominantly from $\mathrm{W}+\mathrm{Jets}$ and QCD events. In hadronic channel it is estimated using fully data-driven technique. In leptonic channel it is estimated using a semi data-driven method where the W+Jets is estimated from MC simulation while the QCD background is estimated from like-sign control region. The remaining backgrounds such as diboson, single top quark, $\mathrm{W} \gamma$ (only in leptonic channel) and SM Higgs production are estimated using MC simulation. The difference in selection, identification, and trigger efficiencies between data and simulation are accounted using different scale factors.

The $\tau$ being much lighter than the Higgs, is produced highly Lorentz boosted. So, we assume its decay products are produced collimated [6]. Under this collinear approximation we build an 


\begin{tabular}{|c|c|c|}
\hline Channel & Yukawa Coupling & 95\% CL Limit \\
\hline$H \rightarrow \mu \tau$ & $\sqrt{\left|Y_{\mu \tau}\right|^{2}+\left|Y_{\tau \mu}\right|^{2}}$ & 0.00143 \\
$H \rightarrow e \tau$ & $\sqrt{\left|Y_{e \tau}\right|^{2}+\left|Y_{\tau e}\right|^{2}}$ & 0.00226 \\
\hline
\end{tabular}

Table 1: 95\% CL observed upper limit on the LFV Yukawa couplings [5]

estimator for the Higgs mass, called the collinear mass, where the $p_{T}$ of the neutrinos is estimated from the projection of the Missing Transverse Momentum(MET) in the direction of the visible $\tau$ decay products. The signal processes are also characterized with final state leptons having a generally harder $p_{T}$ spectrum compared to the background. This helps in reduction of backgrounds like DY and SM Higgs. We also apply cuts on the transverse mass of one of the leptons and the MET in order to achieve better signal siginificance.

A boosted decision tree (BDT) using several kinematic variables as inputs is trained with signal against a combination of backgrounds for each of the channels. The results are extracted using a binned likelihood fit over the BDT output distributions. A cut based analysis which uses collinear mass as the signal variable is used as a cross-check. The limits obtained using just the collinear mass gives us limits which are less sensitive than the BDT but they are still compatible. No significant excess of data is observed over the background. The CLs method [7] is used to set upper limits on $H \rightarrow \mu \tau$ and $H \rightarrow e \tau$ branching fractions at 95\% confidence level (Figure 1). The observed (expected) upper limits are $0.25 \%(0.25 \%)$ and $0.63 \%(0.37 \%)$ for $\mathrm{BR}(H \rightarrow \mu \tau)$ and $\mathrm{BR}(H \rightarrow e \tau)$ respectively while the best fit branching fractions are $0.00 \pm 0.12$ and $0.30 \pm 0.18$

The branching fraction constraints can be translated to constraints on the LFV Yukawa couplings. The 95\% CL limit on these couplings assuming a SM Higgs decay width of $4.1 \mathrm{MeV}$ and mass of $125 \mathrm{GeV}$ are given in Table 1. A previous $2.4 \sigma$ excess observed by CMS in the $H \rightarrow \mu \tau$ channel [8] has been excluded and the LFV Higgs results currently obtained are the most sensitive till date.

\section{Lepton flavor violating decays of neutral Heavy Higgs}

A search for $H \rightarrow \mu \tau$ performed using $8 \mathrm{TeV}$ proton-proton collision data, was recast into limits on the branching fraction times cross-section, for masses up to $300 \mathrm{GeV}$ [9]. Recently, a first direct search for LFV decays of the heavy neutral Higgs boson is performed, which extends the search range up to $900 \mathrm{GeV}$. The search channels are similar to the search for LFV decays of Higgs(125) analysis that is mentioned in the previous section.

The production mode that is targeted is Gluon Gluon Fusion and the signal is modeled assuming narrow width keeping the search as model independent as possible. The search strategy is similar to the cut based approach described in the search for LFV decays of Higgs(125) [5]. Each channel is divided into two categories based on the number of jets in the event namely, 0 -jet and 1-jet, where the jet has a $p_{T}>30 \mathrm{GeV}$ and $|\eta|<4.7$.

The background estimation is done similar to the search for LFV decays of Higgs(125) analysis. The primary difference in this analysis is that the misidentified lepton background in which a jet is misidentified as a lepton is measured only considering the misidentified taus because the misidentified electrons and muons provide a contribution less than $5 \%$ to the misidentified lepton 

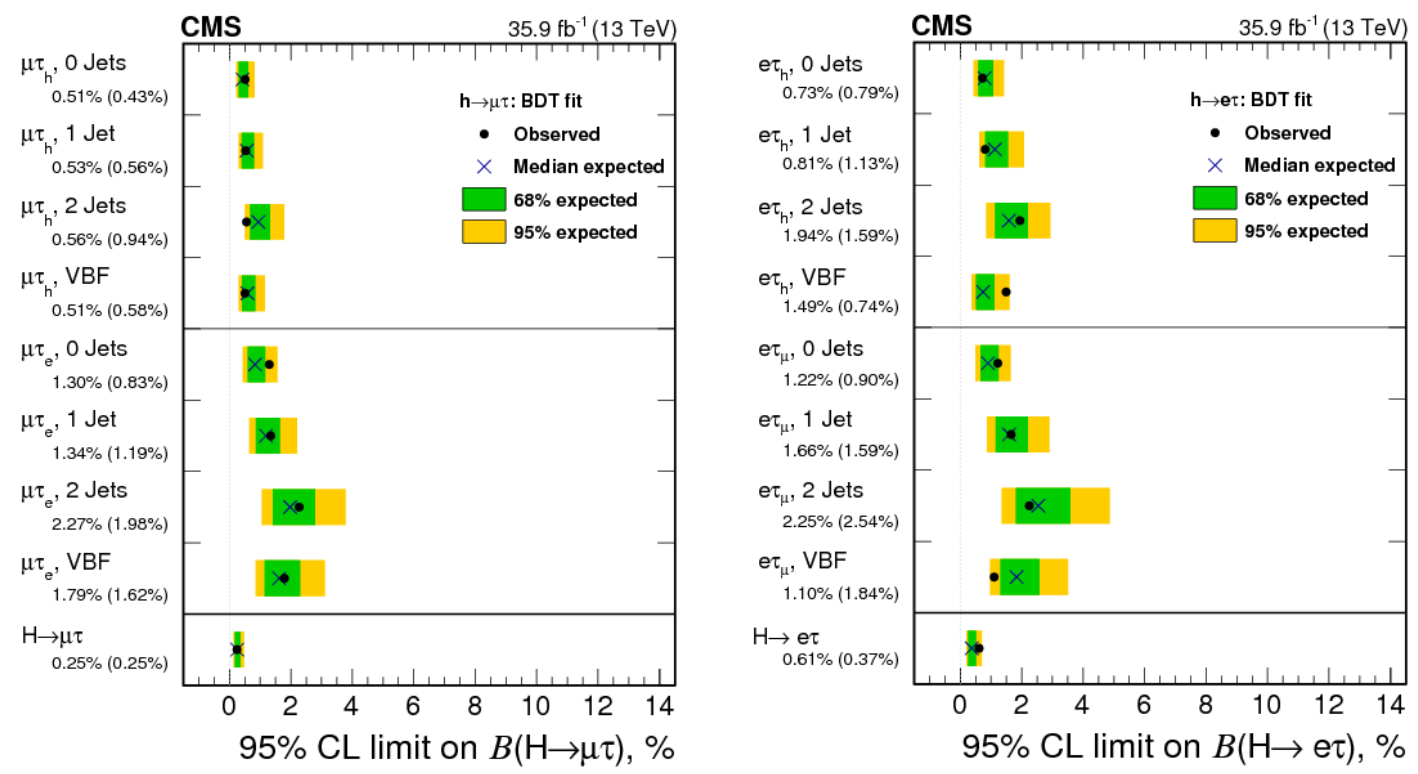

Figure 1: Observed (Expected) $95 \% \mathrm{CL}$ upper limits on $\mathrm{BR}(H \rightarrow \mu \tau)$ and $\mathrm{BR}(H \rightarrow e \tau)[5]$

\begin{tabular}{|c|c|c|}
\hline Channel & Observed & Expected \\
\hline$H \rightarrow \mu \tau(200-900 \mathrm{GeV})$ & 0.0516 to $0.0017 \mathrm{pb}$ & 0.0570 to $0.0021 \mathrm{pb}$ \\
$H \rightarrow e \tau(200-900 \mathrm{GeV})$ & 0.0970 to $0.0023 \mathrm{pb}$ & 0.0880 to $0.0016 \mathrm{pb}$ \\
\hline
\end{tabular}

Table 2: The observed and expected limits on the cross section times the branching fraction of a heavy Higgs boson of mass in the range $200-900 \mathrm{GeV}$ [10]

background. The misidentified lepton background thus estimated is validated in like-sign and $\mathrm{W}$ boson enriched control regions.

To take advantage of the signal topology several kinematic selections are applied to events with two well identified oppositely charged leptons of different flavors. The $p_{T}$ of the leptons is found to be a powerful discriminant variable against most backgrounds. The $\tau$ being much lighter than the Higgs is highly Lorentz boosted, so its decay products are assumed to be collimated [6]. A cut on the azimuthal separation between the $\tau$ lepton decay products can thus help discriminate against several backgrounds. Similarly, a cut on the transverse mass of the $\tau$ and MET can also help in reducing the background contribution.

To get better sensitivity in the entire Higgs mass range two different sets of optimized selection cuts are used. One set is optimized for the low mass region $(200-450 \mathrm{GeV})$, and another set for the high mass region $(450-900 \mathrm{GeV})$. A binned likelihood is then used to fit the collinear mass distributions for the signal and background contributions. No significant excess is observed in any category. The fits are performed per channel and category, which are then combined to set 95\% CL upper limits on heavy Higgs boson production cross section times branching ratio to LFV pairs, $\sigma(g g \rightarrow H) \times B(H \rightarrow \mu \tau)$ and $\sigma(g g \rightarrow H) \times B(H \rightarrow e \tau)$ as a function of $m_{H}$ as can be seen in Figure 2. A profiled likelihood method is used to derive all results assuming asymptotic approximation, and to set upper bounds on the branching fraction the CLs method is used [7]. 

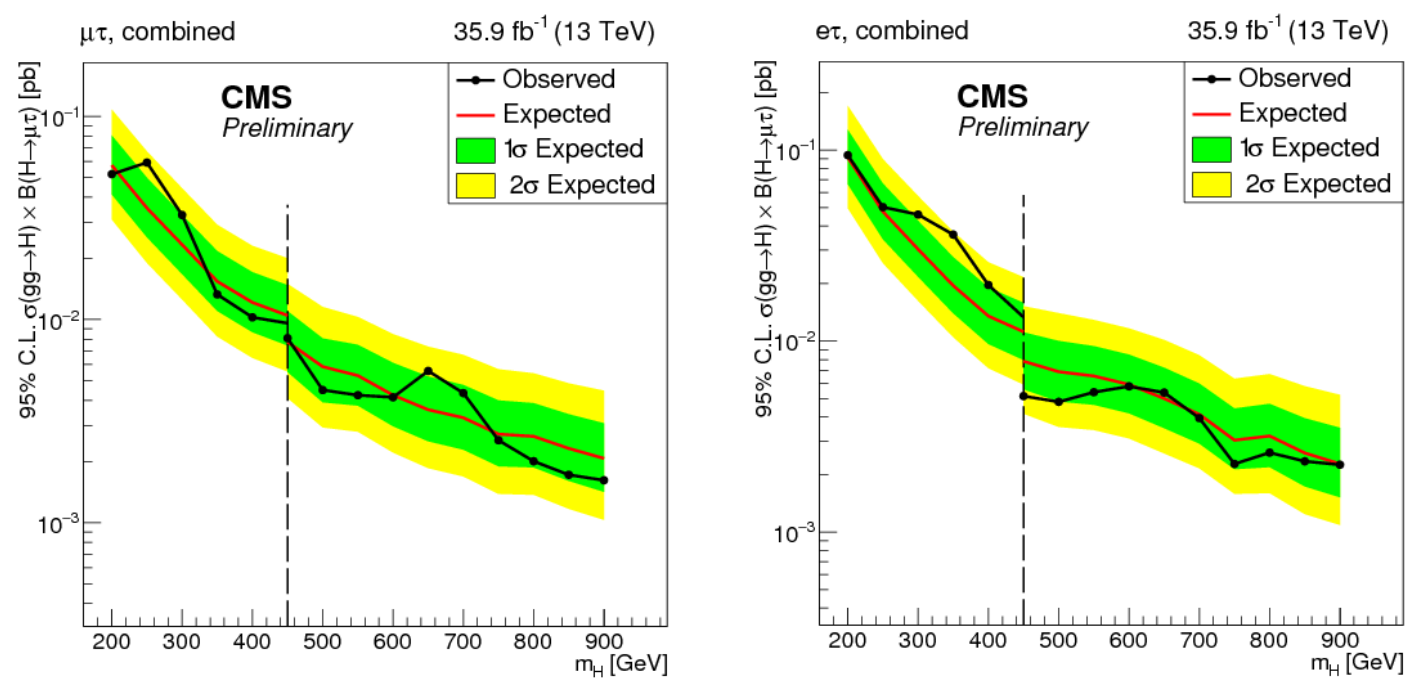

Figure 2: The combined observed and median expected 95\% limits on $\sigma(g g \rightarrow H) \times B(H \rightarrow \mu \tau)$ and $\sigma(g g \rightarrow H) \times B(H \rightarrow e \tau)$ channels [10]

\section{Conclusion}

Higgs boson provides a portal to probe physics beyond the standard model. A direct evidence of new physics can be obtained by searching for Exotic Higgs decays. Two of such searches performed by the CMS collaboration have been presented. No excess is observed in any of the searches discussed. Limits on branching fraction have been set on lepton flavor violating decays of the Higgs and limits on the cross section times the branching fraction have been set for a heavy Higgs boson using data collected in 2016. Using the entire Run II data an updated analysis targeting the lepton flavor violating decays of the Higgs is currently ongoing and CMS expects to set stringent limits on the lepton flavour violating branching fraction.

\section{References}

[1] Serguei Chatrchyan et al. Observation of a New Boson at a Mass of $125 \mathrm{GeV}$ with the CMS Experiment at the LHC. Phys. Lett., B716:30-61, 2012.

[2] Georges Aad et al. Observation of a new particle in the search for the Standard Model Higgs boson with the ATLAS detector at the LHC. Phys. Lett., B716:1-29, 2012.

[3] Georges Aad et al. Measurements of the Higgs boson production and decay rates and constraints on its couplings from a combined ATLAS and CMS analysis of the LHC pp collision data at $\sqrt{s}=7$ and 8 TeV. JHEP, 08:045, 2016.

[4] S. Chatrchyan et al. The CMS Experiment at the CERN LHC. JINST, 3:S08004, 2008.

[5] Albert M Sirunyan et al. Search for lepton flavour violating decays of the Higgs boson to $\mu \tau$ and $\tau \tau$ in proton-proton collisions at $\sqrt{s}=13 \mathrm{TeV}$. JHEP, 06:001, 2018 .

[6] R. Keith Ellis, I. Hinchliffe, M. Soldate, and J. J. van der Bij. Higgs Decay to tau+ tau-: A Possible Signature of Intermediate Mass Higgs Bosons at the SSC. Nucl. Phys., B297:221-243, 1988. 
[7] Alexander L. Read. Presentation of search results: The CL(s) technique. J. Phys., G28:2693-2704, 2002. [,11(2002)].

[8] Vardan Khachatryan et al. Search for Lepton-Flavour-Violating Decays of the Higgs Boson. Phys. Lett., B749:337-362, 2015.

[9] Malte Buschmann, Joachim Kopp, Jia Liu, and Xiao-Ping Wang. New Signatures of Flavor Violating Higgs Couplings. JHEP, 06:149, 2016.

[10] CMS Collaboration. Search for lepton flavour violating decays of neutral heavy Higgs boson to $\mu \tau$ and $\tau$ in proton-proton collisions at $\sqrt{s}=13 \mathrm{TeV} .2019$. 